\title{
Assessing the Risks of Trading Strategies Using Acceptability Indices
}

\author{
Masimba E. Sonono ${ }^{1}$, Hopolang P. Mashele ${ }^{2}$ \\ ${ }^{1}$ Unit for Business Mathematics and Informatics, North-West University, Potchefstroom, South Africa \\ ${ }^{2}$ Centre for Business Mathematics and Informatics, North-West University, Potchefstroom, South Africa \\ Email: 23756144@nwu.ac.za, phillip.mashele@nwu.ac.za
}

Received August 4, 2013; revised September 30, 2013; accepted October 11, 2013

Copyright (C) 2013 Masimba E. Sonono, Hopolang P. Mashele. This is an open access article distributed under the Creative Commons Attribution License, which permits unrestricted use, distribution, and reproduction in any medium, provided the original work is properly cited.

\begin{abstract}
The paper looks at the quantification of risks of trading strategies in incomplete markets. We realized that the no-arbitrage price intervals are unacceptably large. From a risk management point of view, we are concerned with finding prices that are acceptable to the market. The acceptability of the prices is assessed by risk measures. Plausible risk measures give price bounds that are suitable for use as bid-ask prices. Furthermore, the risk measures should be able to compensate for the unhedgeable risk to an extent. Conic finance provides plausible bid-ask prices that are determined by the probability distribution of the cash flows only. We apply the theory to obtain bid-ask prices in the assessment of the risks of trading strategies. We analyze two popular trading strategies-bull call the spread strategy and bear call spread strategy. Comparison of risk profiles for the strategies is done between the Variance Gamma Scalable Self Decomposable model and the Black-Scholes model. The findings indicate that using bid-ask prices compensates for the unhedgeable risk and reduces the spread between bid-ask prices.
\end{abstract}

Keywords: Conic Finance; Coherent Risk Measure; Acceptability Indices; Incomplete Markets; Bid-Ask Prices; Continuous Time Models

\section{Introduction}

The paper focuses on the quantification of risks of trading strategies, particularly when the market is incomplete. The incompleteness of the market gives rise to many martingales, each of which produces a no-arbitrage price. Thus there is no exact replication so as to obtain a unique price. Furthermore, the no-arbitrage price intervals are unacceptably large. From a risk management point of view, we are concerned with finding the prices which are acceptable. The acceptability of these prices is assessed by risk measures.

In the financial literature, two major classes of risk measures have gained ground in assessing the risks of financial positions. Foremost, we have coherent measures introduced by [1]. Since then, the theory of coherent risk measures has been applied to several problems in finance. Secondly, there is the grounding work of [2], in which they proposed a new class of performance measures known as acceptability indices. The acceptability indices can be considered as an extension of coherent risk measures. Under the acceptability framework, a fi- nancial position is acceptable if its distribution function withstands high levels of stress, or in other words, a stressed sampling of the financial position has a positive expectation. In this paper, our contribution is assessing the risk profiles of trading strategies using the acceptability framework.

The rest of the paper is organized as follows: Section 2 looks at the problem of pricing in incomplete markets. Section 3 gives an overview of risk measures and presents new acceptability indices based on the family of distortion functions. Section 4 presents a brief detail on conic finance and provides closed form expressions for the bid-ask prices. Section 5 presents the models that are used in this work. Section 6 presents numerical tests on assessing the risks of two trading strategies. Section 7 is the conclusion.

\section{Problem of Pricing in Incomplete Markets}

We start by motivating the problem through explaining the mathematical structure of good deal bounds by [3], also found in [4]. The good deal bounds determine the 
range of values of a risky position payoff. Let $R$ be the set of replicable payoffs, $\Pi(Y)$ be the market price to replicate a payoff $Y \in R$, and $A$ be an acceptance set of payoffs that are acceptable to the situation. The lower good deal bound for a payoff $X$ is:

$$
b(X)=\sup _{Y \in R}\{-\Pi(Y) \mid Y+X \in A\} .
$$

This payoff might be interpreted as a bid price. Equation (1) tells us that if $X$ can be bought for less than $b(X)$, then there is a $Y$ that can be bought for $\Pi(Y)$ such that a cost $b(X)+\Pi(Y)<0$. The upper good deal bound, which might be interpreted as the ask price for $X$, is given by:

$$
a(X)=-b(-X)=\inf _{Y \in R}\{\Pi(Y) \mid Y-X \in A\} .
$$

Equation (2) tells us that selling $X$ or buying $-X$ yields the same effect. The interpretation of $-b(X)$ is the cost that renders $X$ to be acceptable. As [5] propose: any valuation principle that gives price bounds induces a risk measure and vice versa. The acceptance set $A$ must include the set of riskless payoffs, $\{Z \mid Z \geq 0\}$, which is the acceptance set that generates no-arbitrage bounds. The set $A$ does not intersect with the set $\{Z \mid Z<0\}$ of pure losses. The acceptance set $A$ must be consistent with market prices, $\Pi$, or arbitrage occurs.

Now, an incomplete market is one in which there are many martingale measures $Q$. The price bounds in Equations (1) and (2) form an interval of arbitrage-free prices for $X$ :

$$
I=\left(\inf _{Q \in \mathcal{M}} E^{Q}[X], \sup _{Q \in \mathcal{M}} E^{Q}[X]\right)
$$

where $\mathcal{M}$ is a set of equivalent martingale measures. The problem with the interval of the arbitrage-free prices for $X$ is that it is usually too wide for the no-arbitrage bounds to serve as useful bid-ask prices.

In practice, derivatives traders are aware of the incompleteness of the markets and after making trades on certain positions, they are not able to hedge away all the risk. Instead, they must bear the risk associated with the trade. To cover their business expenses and to earn compensation for bearing the risk they are not able to hedge, traders establish bid-ask intervals around the expected discounted payoff.

Now, in constructing the bid-ask prices, the difficulty posed by incomplete markets is very significant because of adverse selection. For instance, if the ask price is too high, few potential investors will be willing to pay so much and the result is foregone profits. If the ask price is too low, the resulting trade is bad for a trader and entails likely losses. So, to ensure that trades made at bid and ask prices are beneficial, it helps to use methods that produce bounds for the prices that are suitable for use as bid-ask prices and are adequate to minimize unhedgeable risk to an extent. In the process, we will be able to quantify risk since any valuation method that yields price bounds also induces a risk measure [5].

\section{Risk Performance Measures}

In this section, we give a brief overview of the risk measures. In general, a risk measure, $\rho: X \rightarrow \mathbb{R}$, is a functional that assigns a numerical value to a random variable representing an uncertain payoff.

\subsection{Coherent Risk Measure}

\section{Definition Coherent Risk Measure}

A risk measure $\rho$ is coherent if it satisfies the following axioms:

- Translation Invariance:

$$
\rho(X+\alpha r)=\rho(X)-\alpha,
$$

for all $X \in \mathcal{G}, \alpha \in \mathbb{R}$.

- Monotonicity: $\rho(X) \leq \rho(Y)$ if $X \geq Y$ a.s.

- Positive Homogeneity:

$$
\rho(\lambda X)=\lambda \rho(X),
$$

for $\lambda \geq 0$.

- Subadditivity:

$$
\rho(X+Y) \leq \rho(X)+\rho(Y),
$$

for all $X, Y \in \mathcal{G}$

- Relevance: $\rho(X)>0$ if $X \leq 0$ and $X \neq 0$.

The last property is included although it is not a determinant of coherency. Translation invariance axiom implies that by adding a fixed amount $\alpha$ to the initial position and investing it in a reference instrument, the risk $\rho(X)$ decreases by $\alpha$. The monotonicity axiom postulates that if $X(\omega)>Y(\omega)$ for every state of nature $\omega$, $Y$ is more risk because it has higher risk potential.

The positive homogeneity axiom implies that risk linearly increases with size of the position, that is to say that the size of the risk of a position should scale with the size of the position. This is just a natural requirement, though this condition may not be satisfied in the real world since markets may be illiquid. The subadditivity axiom implies that the risk of a portfolio is always less than the sum of the risks of its subparts. This axiom ensures that diversification decreases the risk.

According to the basic representation theorem proved by [1] for a finite $\Omega$, any coherent risk measure admits a representation of the form:

$$
\rho(X)=-\inf _{Q \in \mathcal{D}} E^{Q}[X]
$$

with a certain set $\mathcal{D}$ of probability measures with respect to $P$. A cash flow $X$ is acceptable if it has negative risk, that is $\rho(X)<0$. 


\subsection{Acceptability Indices}

Cherny and Madan, defined a subclass of risk measures called acceptability indices, defined formally as:

\subsection{Definition. Index of Acceptability}

The acceptability index is as a mapping $\alpha$ from the set of bounded random variables to the extended half-line $[0, \infty]$. The index satisfies the following four properties:

\section{- Monotonicity}

If $Y$ dominates $X$, that is $X \leq Y$, then

$$
\alpha(X) \leq \alpha(Y) .
$$

\section{- Scale invariance}

$\alpha(X)$ stays the same when $X$ is scaled by a positive number, that is $\alpha(c X)=\alpha(X)$ for $c>0$.

\section{- Quasi-concavity}

If $\alpha(X) \geq Y$ and $\alpha(Y) \geq Y$, then

$$
\alpha(\lambda X+(1-\lambda) Y) \geq Y,
$$

for any $\lambda \in[0,1]$.

\section{- Fatou Property (Convergence)}

Let $\left\{X_{n}\right\}$ be a sequence of random variable. $\left|X_{n}\right| \leq 1$ and $X_{n}$ converges in probability to a random variable $X$. If $\alpha\left(X_{n}\right) \geq x$, then $\alpha(X) \geq x$.

The acceptability indices are constructed by replacing the cumulative distribution function of $X, F_{X}(x)$, by a risk adjusted distribution, $\Psi_{X}\left(F_{X}(x)\right)$. The corresponding risk measure is the negative expectation of the zero cost cash flow under the distorted distribution function:

$$
\rho_{\gamma}(X)=-\int_{\mathbb{R}} x \mathrm{~d}\left(\Psi_{\gamma}\left(F_{X}(x)\right)\right), \gamma \in \mathbb{R}_{+}
$$

where $\Psi_{\gamma}$ is a family of concave distortion functions on $[0,1]$ increasing pointwise in the stress level parameter $\gamma$. A higher value of $\gamma$ results in severe distortion of the distribution function of $X$. Then, the acceptability index, $\alpha(X)$, is the largest stress level $\gamma$ such that the expectation of $X$ remains positive under the distortion or in other words the distorted cash flow remains acceptable:

$$
\alpha(X)=\sup \left\{\gamma \in \mathbb{R}_{+}: \rho_{\gamma}(X) \leq 0\right\} .
$$

Cherny and Madan introduced four acceptability indices based on the family of distortion functions which are namely: AIMIN, AIMAX, AIMINMAX, AIMAXMIN.

- AIMIN is the largest number $x$ such that the expectation of the minimum of $x+1$ draws from cash flow distribution is still positive. Let

$$
Y=\operatorname{law} m i n X_{1}, \cdots, X_{x+1},
$$

where $X_{1}, \cdots, X_{x+1}$ are independent draws from $X$. The concave distortion function is given by:

$$
\Psi_{x}(y)=1-(1-y)^{x+1}, x \in \mathbb{R}^{+}, y \in[0,1]
$$

- AIMAX constructs a distribution from which one draws numerous times and takes the maximum to get the cash flow distribution being evaluated. Let,

$$
\max \left\{Y_{1}, \cdots, Y_{x+1}\right\} \stackrel{\text { law }}{=} X
$$

where $Y_{1}, \cdots, Y_{x+1}$ are independent draws of $Y$. The concave distortion function is given by:

$$
\Psi_{x}(y)=y^{\frac{1}{x+1}}, x \in \mathbb{R}^{+}, y \in[0,1]
$$

- AIMAXMIN is constructed by first using the MINVAR and then followed by the MAXVAR to create worst case scenarios.

Let

$$
\max \left\{Y_{1}, \cdots, Y_{x+1}\right\} \stackrel{\text { law }}{=} \min \left\{X_{1}, \cdots, X_{x+1}\right\},
$$

where $X_{1}, \cdots, X_{x+1}$ are independent draws of $X$ and $Y_{1}, \cdots, Y_{x+1}$ are independent draws of $Y$. Combining the MINVAR and MAXVAR, we have the distortion function:

$$
\Psi_{x}(y)=\left(1-(1-y)^{x+1}\right)^{\frac{1}{x+1}}, x \in \mathbb{R}^{+}, y \in[0,1]
$$

- AIMAXMIN is constructed by first using the MAXVAR and then followed by the MINVAR to create worst case scenarios. Let

$$
\begin{aligned}
& Y \stackrel{\text { law }}{=} \min Z_{1}, \cdots, Z_{x+1}, \\
& \max Z_{1}, \cdots, Z_{x+1} \stackrel{\text { law }}{=} X,
\end{aligned}
$$

where $Z_{1}, \cdots, Z_{x+1}$ are independent draws of $Z$. Combining the MINVAR and MAXVAR, we have the distortion function:

$$
\Psi_{x}(y)=1-\left(1-y^{\frac{1}{x+1}}\right)^{x+1}, x \in \mathbb{R}^{+}, y \in[0,1]
$$

The acceptability indices are more plausible in assessing the risks of financial positions. The acceptability indices have been used heavily in the theory of conic finance, which we review next.

\section{Conic Finance Theory}

We look at the principles of conic finance as set out in [6]. The market serves a passive counterparty accepting the opposite side of zero cost trades proposed by market participants. The departure of conic finance from the traditional one price economy is that trade now depends on the direction of trade, with the market buying at bid price and selling at ask price. Cash flows to trade are modeled as bounded random variables on a fixed probability space $(\Omega, \mathcal{F}, P)$ for a base probability measure selected by the economy.

Now, for a risk with a cash flow outcome denoted by 
the random variable $X$ with a distribution $F(x)$ at a fixed period, we develop bid-ask prices at which the cash flow is bought and sold such that the net cash flow is an acceptable risk. The set of acceptable risks is defined by a convex cone of random variables that contains the non-negative cash flows. [1] showed that any acceptable set (cone) $\mathcal{A}$ of acceptable risks, there exists a convex set $\mathcal{M}$ of probability measures $Q \in \mathcal{M}, Q$ equivalent to $P$, with the property that $X \in \mathcal{A}$ if and only if:

$$
E^{Q}[X] \geq 0, \text { all } Q \in \mathcal{M} \text {. }
$$

The acceptability of a cash flow can then be completely determined by its distribution function. Acceptability of cash flows is linked to positive expectation via concave distortion. So for some concave distribution function $\Psi(u), 0 \leq u \leq 1$ the cash flow distribution function $F(x)=P(X \leq x)$ is acceptable if:

$$
\int_{-\infty}^{\infty} x \mathrm{~d} \Psi(F(x)) \geq 0 .
$$

[6] show that the bid price, $b(x)$, for the cash flow $X$ is given by:

$$
b(x)=\int_{-\infty}^{\infty} x \mathrm{~d} \Psi(F(x)) \geq 0=\inf _{Q \in \mathcal{M}} E^{Q}[X],
$$

and the ask price is given by:

$$
a(x)=-\int_{-\infty}^{\infty} x \mathrm{~d} \Psi(1-F(-x)) \geq 0=\sup _{Q \in \mathcal{M}} E^{Q}[X] .
$$

The bid and ask prices for call and put options can be obtained by using closed formulas which are obtained on integration by parts. Let $S$ be the random variable at time $T$ of an underlying asset. The call option $C=(S-K)^{+}$and put option $P=(K-S)^{+}$, where $K$ is the strike price. The following are the closed bid and ask prices expressions:

$$
\begin{gathered}
a_{\gamma}(C)=\int_{K}^{\infty} \Psi^{\gamma}\left(1-F_{S}(x)\right) \mathrm{d} x, \\
b_{\gamma}(C)=\int_{K}^{\infty}\left(1-\Psi^{\gamma}\left(F_{S}(x)\right)\right) \mathrm{d} x, \\
a_{\gamma}(P)=\int_{0}^{K} \Psi^{\gamma}\left(F_{S}(x)\right) \mathrm{d} x, \\
b_{\gamma}(P)=\int_{0}^{K}\left(1-\Psi^{\gamma}\left(1-F_{S}(x)\right)\right) \mathrm{d} x .
\end{gathered}
$$

$F_{s}$ is the distribution function of $S$ and is important because the bid and ask prices are determined completely by this distribution.

\section{Continuous Time Models for Option Pricing}

This section looks at the models that are used for option pricing. It is acknowledged that the relatively most liquid traded assets with market information are quoted vanilla options. In practice, trades mark to market their models to quoted vanilla options before they can price nonquoted options. As a result, this has led to demands for models that are capable of synthesizing the surface of vanilla options. It is well known that the geometric Brownian model is not capable of synthesizing the surface of vanilla options, although it remains a standard quoting model in the markets. Improvements on this model are offered by Lévy processes, which were found to be successful in synthesizing across strikes for a given maturity. The following is a brief overview of the models.

\subsection{Black-Scholes Model}

The log-normal process models continuously compounded returns using the general Brownian motion so that:

$$
X(t)=v t+\sigma W(t)
$$

where $W(t)$ is a standard Weiner process, $v$ is the instantaneous drift and $\sigma$ is the instantaneous volatility of returns. The stochastic differential equation of the stock price is:

$$
\mathrm{d} S(t)=S(t)(\mu \mathrm{d} t+\sigma \mathrm{d} W(t)),
$$

where $\mu$ is the growth rate of the stock and is related to $v$ as follows $v=\mu-1 / 2 \sigma^{2}$. The stochastic differential equation can be solved to give the following dynamics of the stock price:

$$
S(t)=S(0) \exp \left\{\left(\mu-\frac{1}{2} \sigma^{2}\right) t+\sigma W(t)\right\} .
$$

The characteristic function for the logarithm of the stock price is:

$$
E\left[\mathrm{e}^{i u \ln (S(t))}\right]=\exp \left\{i u\left[\ln S(0)+\left(\mu-\frac{1}{2} \sigma^{2}\right)\right] t-\frac{1}{2} \sigma^{2} u^{2} t\right\}
$$

\subsection{Variance Gamma Model}

[7] define a Variance Gamma process, $X(t, v, \sigma, \theta)$, as a time changed Brownian motion as follows:

$$
X(t, v, \sigma, \theta)=\theta \gamma(t)+\sigma W(\gamma(t)),
$$

where $\gamma(t)$ is a Gamma process with parameters $a$ and $b$, that is, $\gamma(t) \sim \operatorname{Gamma}(a t, b)$ where the gamma probability density function $\gamma(a, b)$ is given by:

$$
f_{\gamma}(x, a, b)=\frac{b^{a} x^{a-1}}{\Gamma(a)} \mathrm{e}^{-b x}, x>0 .
$$

$\theta$ and $\sigma$ are respectively the instantaneous drift and volatility and $W(t)$ is a standard Brownian motion. The Variance Gamma process uses a gamma process to time change a Brownian motion. The density function of 
a Variance Gamma process is known in closed form and requires the computation of the modified Bessel function of the second kind which can be time consuming. Thus we resort to using the characteristic function, which is found by the conditioning on the jump $\gamma(t)$ as in many Lévy processes and is given by:

$$
\Phi_{X(t)}(u)=\left(1-i u v \theta+\frac{1}{2} u^{2} v \sigma^{2}\right)^{-t / v}
$$

The dynamics of the stock price are given by:

$$
S(t)=S(0) \exp \{(\mu+\omega) t+X(t, v, \sigma, \theta)\},
$$

where $\mu$ is the instantaneous expected return of the stock evaluated at calendar time and $\omega$ is a compensator term The characteristic function for the logarithm of stock price is:

$$
E\left[\mathrm{e}^{i u \ln (S(t))}\right]=\exp \{i u[\ln S(0)+(\mu+\omega) t]\} \Phi_{X(t)}(u) .
$$

The compensator term can be found from the characteristic function and is given by:

$$
\omega=\frac{-1}{t} \ln \left(\Phi_{X(t)}(-i)\right) \text {. }
$$

\subsection{Variance Gamma Scalable Self Decomposable (VGSSD) Model}

Sato process model was first introduced by [8]. The Sato process was shown to be effective in synthesizing many options on numerous underliers at the same time. The idea behind the model was to construct stochastic processes that had inhomogeneous independent increments from Lévy processes with homogeneous independent increments such that the higher moments are constant over the time horizon.

The starting point for the construction of the Sato model is the self-decomposable law. Loosely speaking, the self-decomposable law describes random variables that decompose into the sum of a scaled down version of themselves and an independent residual term. The scaling property means the distribution of increments of various time scales can be obtained from that of other time scale by rescaling the random variable at that time scale. Thus the distribution at larger time scales are derived from those at smaller time scales, which are easier to estimate as the data are sufficient. [9] proposed that the self-decomposable law is associated with the unit time distribution of self-similar additive process whose increments are independent, but not necessarily stationary.

It is known that stock prices are moved by many pieces of information. If the pieces of information are considered as a sequence of independent random variables $\left(Z_{i}: i=1,2, \cdots\right)$, then the price changes are con- sequences of the impacts from all $Z_{i}$. Now, let $S_{n}=\sum Z_{i}$ denote their sum. Suppose that there exist centeriffg constants $c_{n}$ and scaling constants $b_{n}$ such that the distribution of $b_{n} S_{n}+c_{n}$ converges to the distribution of the random variable $X$, which belongs to a family law "class $L$ ". Then the random variable $X$ is said to have the class L property. So, the price change over the time horizon is the outcome of many independent random variables which can be approximated as a random variable $X$ that has the law of "class $L$ ". [10] define the self-decomposable law as follows.

\subsection{Definition Self Decomposable Law}

A random variable $X$ is self-decomposable if for all $c \in(0,1)$,

$$
X \stackrel{\text { law }}{=} c X+X^{c},
$$

where $X^{c}$ is a random variable independent of $X$.

The self-decomposable random variable $X$ can be decomposed into a partial of itself and another independent random variable. [10] also shows that one may associate with such a self-decomposable law at unit time a process with independent but inhomogeneous increments by defining the marginal law of the process at time points $t$ upon scaling the law at unit time. Therefore we have that:

$$
X(t)=t^{\gamma} X, t>0 .
$$

Thus we can study the price changes easily using self-decomposable laws, which are easier to handle than class L.

Self-decomposable laws are an important sub-class of the class of infinitely divisible laws [11]. The characteristic function of the self-decomposable laws has the form (see [10])

$$
E\left[\mathrm{e}^{i u x}\right]=\exp \left\{i r u-\frac{1}{2} \sigma^{2} u^{2}+\int_{R}\left(\mathrm{e}^{i u x}-1-i u x 1_{|x| \leq 1} \frac{g(x)}{|x|} \mathrm{d} x\right)\right\},
$$

where $r, \sigma$ are constants, $\sigma^{2} \geq 0$,

$$
\int_{R}\left(|x|^{2} \wedge 1\right) \frac{g(x)}{|x|} \mathrm{d} x<\infty,
$$

and $g(x)$ is an increasing function when $x<0$ and decreasing function when $x>0$. An infinitely divisible law is self-decomposable if the corresponding Lévy density has the form

$$
\frac{g(x)}{|x|}
$$

where $g(x)$ is increasing for negative $x$ and decreasing for positive $x$.

The dynamics of the stock price is defined as: 


$$
S(t)=S(0) \exp \{r(t)+X(t)+\omega(t)\},
$$

where $\omega(t)$ is a compensator term. The Sato process used in this work is the one constructed from the variance gamma process and is known as the Variance Gamma Scalable Self Decomposable (VGSSD) process. The variance gamma process is defined by time changing an arithmetic Brownian motion with drift $\theta$ and volatility $\sigma$ by an independent gamma process with unit mean rate and variance rate $v$. Let $G(t ; v)$ be the gamma process, then the variance gamma process is written as:

$$
X_{V G}(t, \sigma, v, \theta)=\theta G(t ; v)+\sigma W(G(t ; v)),
$$

where $W(t)$ is an independent standard Brownian motion.

The gamma process is an increasing pure jump Lévy process with independent identically distributed increments over regular non overlapping intervals of length $h$ that are gamma distributed with density $f_{h}(g)$ where:

$$
f_{h}(g)=\frac{g^{\frac{h}{v}-1} \mathrm{e}^{-\frac{g}{v}}}{v^{\frac{h}{v}} \Gamma\left(\frac{h}{v}\right)}, g>0 .
$$

The VGSSD is constructed from the variance gamma process by defining the scaled stochastic process $X(t)$ such that it is equal in law to $t^{\gamma} X_{V G}(1)$ where $X_{V G}(1)$ is a variance gamma random variable at unit time. It follows that the characteristic function of $X(t)$ is given by [7]

$$
\Phi_{X(t)}(u)=\Phi_{X_{V G}(1)}=\left(1-i u t^{\gamma} v \theta+\frac{1}{2} u^{2} t^{2 \gamma} v \sigma\right) .
$$

Since the VGSSD is a scaled stochastic process, its higher moments remain constant over time.

\section{Numerical Tests}

Next, focus is shifted to analyzing the risk profiles of option investing strategies. We examine two option strategies which are namely bull call spread strategy and bear call spread strategy. We determine the maximum risk, maximum reward and breakeven price for each of the strategies. Comparison of risk profiles is done between the VGSSD model and the Black-Scholes model. The Black-Scholes model is considered here since it is the one that is mostly used by industrial practioners. So, the Black-Scholes is a proxy for market prices. The theory of conic finance provides bid-ask prices, which depend on the risk appetite of investors. For evaluation of bid-ask prices, we use acceptability indices based on the MAXMINVAR. The options used in the strategies are of
European type and are applied to Single Stocks Futures (SSF) options offered in the South African financial markets.

A bull call spread is a bullish strategy formed by buying an "in-the-money call option" (lower strike) and selling "out-of-the-money" (higher strike). Both call options must be on the same underlying and expiration date. The strategy's net effect is to bring down the cost and breakeven (long call strike price + net debt) on a buy call (long call) strategy.

A bear call spread is a bearish strategy formed by buying an "out-of-the-money" call option (higher strike) and selling an "in-the-money" call option (lower strike). Both call options must be on the same underlying security and expiration date. The strategy's concept is to protect the downside of the sold call option by buying a call option of higher strike price. Then, the investor receives a net credit since the call option which has been bought has a higher strike price than the sold option. The breakeven will be the sum of the strike price of the short call option plus the premium received.

For numerical illustration purposes, we used names of two large South African banks-ABSA and Standard Bank. Note that, the illustrations do not pertain to any real positions on the banks. The bid-ask prices were computed at various theoretical prices of the underlying on the expiration date. The 3-month JIBAR is used as a proxy for the risk-free interest rate. To realize model calibration, we need market prices. Simulated data set of bid-ask options at different strikes maturing on the same date were generated using the models introduced in the previous Section.

The illustrations that follow merely suggest what an investor can do given the different risk appetites on an investor. The illustrations are implemented at stress (risk) level of $0.01,0.05$ and 0.10 .

\subsection{Bull Call Spread Risk Profile}

\subsubsection{Scenario}

An investor owns 100 shares in ABSA Bank (ASAQ), which in early July are trading at a Single Stock Future (SSF) fair value of R140. The investor believes the market will be bullish in the coming 6 months and decided to create a bull call spread. So the investor buys a DEC ASAQ 140 call option and sells a DEC ASAQ call option with a higher strike price, so as to create the bull call spread strategy. The concern for the investor is on the appropriate higher strike which can create an attractive strategy.

1) At different stress (risk) levels, the investor determines the bid-ask prices for the range of strike prices.

2) The investor analyzes the risk profiles at each strike price choice so as to create an appropriate trade.

3) The investor finally assesses the performance of the 
strategy, given a range of possible values of the underlying at expiration for the appropriate strike price from step 2 at a stress level of 0.01 .

In addition, the investor gathers the following information:

$$
\begin{gathered}
\text { 3month JIBAR rate }=5.01 \% \\
\text { Time to expiration }=6 / 12 \mathrm{yr} \\
\text { Dividend yield }=0 \% \text { (assumption). }
\end{gathered}
$$

In order to create the strategy appropriately, the investor implemented the following steps.

\section{1) Bid-Ask Prices at Different Stress $(\gamma)$ Levels}

The calibrated parameters used for this strategy are $\sigma=0.240$ in the Black-Scholes model and

$$
\sigma=0.226, \theta=-0.131, v=0.08, \gamma=0.480
$$

in the VGSSD model. An attractive bull call spread is created when an investor buys a lower strike call and sells a higher strike call. In the scenario presented above, the investor has the choices shown in Table 1. Table 2 shows the bid-ask prices for the options using both the Black-Scholes model and VGSSD model.

\section{2) Risk Profile Analysis}

Next, we look at the risk profiles for each of the choices using bid-ask prices provided in step 1 at a stress level of 0.01. Under the Black-Scholes model, an attractive strategy can be created by choice 4) as shown in Table 3. The reason is that the risk and breakeven point is lower whilst maximum reward and maximum Return on Investment (ROI) are high enough to be attractive.

Also under the VGSSD model, an attractive strategy can be created using choice 4) as shown in Table 4 . The reason being again that the risk and breakeven is lower whilst the maximum reward and maximum Return on Investment (ROI) are high enough to be attractive.

\section{3) Scenario Analysis at the Expiration Date}

After choosing an attractive choice from step 2, we now look at the profit/loss of the strategy at expiration for a range of prices for the underlying. We compare the profit/loss under the two models-Black-Scholes model and VGSSD model. Table 5 shows the profit/loss of the strategy under the two models. Figure 1 shows the plot of the profit/loss of the strategy for a range of prices of the underlying at expiration. From Figure 1, it can be observed that the breakeven point is lower using the VGSSD model than the Black-Scholes model. A lower breakeven point is ideal for a strategy which intends to reduce risk.

\subsubsection{Comment on the Strategy}

The spread was observed to be lower in the VGSSD model than in the Black-Scholes model. Reduced spread can minimize the unhedgeable risk, which can be a major
Table 1. Bull call spread investor choices.

\begin{tabular}{ccl}
\hline Step 1 & Long Call & Buy R140 Strike Call \\
\hline Step 2 & 1) Short Call & Sell R141 Strike Call \\
Or & 2) Short Call & Sell R142 Strike Call \\
Or & 3) Short Call & Sell R143 Strike Call \\
Or & 4) Short Call & Sell R144 Strike Call \\
Or & 5) Short Call & Sell R145 Strike Call \\
\hline
\end{tabular}

boost for option trading strategies. As a result, the cost of trade is lowered as the sold options can offset the cost of the bought option. In conclusion, the strategy becomes less risky in terms of lower risk and lower breakeven point but offers limited potential reward, which can still be highly attractive.

\subsection{Bear Call Spread Risk Profile}

\subsubsection{Scenario}

In early July an investor believes the SSF fair price of Standard Bank (SBKQ) is going to fall from the current levels of R120 to around R117.50. The investor wants to create an attractive bear call spread. So the investor writes a SEP SBKQ 119 call option and buys a higher SEP SBKQ strike call, so as to create a bear call strategy. A little bit of concern to the investor is on the appropriate higher strike to choose so as to create an attractive strategy.

1) Now at different stress (risk) levels, the investor determines the bid-ask prices for the range of higher strike prices.

2) The investor analyzes the risk profiles at each of strike price choices so as to create an appropriate trade.

3) Finally, the investor accesses the performance of the strategy for the appropriate strike price in step 2 at a stress level of 0.01 given a range of possible values of the underlying at expiration.

1) Bid-Ask Prices at Different Stress $(\gamma)$ Levels

The calibrated parameters used for this strategy are $\sigma=0.306$ in the Black-Scholes model and

$$
\sigma=0.285, \theta=-0.070, v=0.060, \gamma=0.510
$$

in the VGSSD model. An attractive bear call spread is created when an investor sells a lower strike call and buys a higher strike call. In the scenario presented here, the investor has the choices shown in Table 6. Table 7 shows the bid-ask prices for the options using both the Black-Scholes model and VGSSD model.

\section{2) Risk Profile Analysis}

We now look at the risk profiles for each of the choices using bid-ask prices provided in step 1 at a stress level of 0.01 . In Table 8 a potential strategy can be created using a strike which provides reduced risk and a lower breakeven point. In addition, the gain on this strategy is the net credit received upon entering the trade. As 
Table 2. Bull call spread bid-ask prices at different stress levels.

\begin{tabular}{|c|c|c|c|c|c|c|c|c|c|c|}
\hline \multirow{2}{*}{$\begin{array}{l}\text { Stress } \\
\text { Level }\end{array}$} & \multicolumn{5}{|c|}{ Black-Scholes Model } & \multicolumn{5}{|c|}{ VGSSD Model } \\
\hline & $\mathbf{S}$ & $\mathbf{K}$ & Bid & Ask & Spread & $\mathrm{S}$ & $\mathbf{K}$ & Bid & Ask & Spread \\
\hline \multirow[t]{5}{*}{0.01} & 140 & 140 & 11.05 & 11.83 & 0.78 & 140 & 140 & 11.04 & 11.77 & 0.73 \\
\hline & & 142 & 10.07 & 10.81 & 0.74 & & 142 & 10.04 & 10.73 & 0.69 \\
\hline & & 143 & 9.62 & 10.33 & 0.71 & & 143 & 9.59 & 10.26 & 0.67 \\
\hline & & 144 & 9.16 & 9.85 & 0.69 & & 144 & 9.15 & 9.79 & 0.64 \\
\hline & & 145 & 8.71 & 9.37 & 0.66 & & 145 & 8.70 & 9.32 & 0.62 \\
\hline \multirow[t]{5}{*}{0.05} & 140 & 140 & 10.50 & 14.59 & 4.09 & 140 & 140 & 10.49 & 14.28 & 3.79 \\
\hline & & 141 & 10.03 & 14.00 & 3.79 & & 141 & 10.01 & 13.68 & 3.67 \\
\hline & & 142 & 9.56 & 13.40 & 3.84 & & 142 & 9.53 & 13.09 & 3.56 \\
\hline & & 143 & 9.09 & 12.80 & 3.71 & & 143 & 9.07 & 12.52 & 3.45 \\
\hline & & 144 & 8.67 & 12.28 & 3.61 & & 144 & 8.66 & 12.01 & 3.35 \\
\hline \multirow[t]{6}{*}{0.10} & 140 & 140 & 9.85 & 18.45 & 8.60 & 140 & 140 & 9.81 & 17.67 & 7.86 \\
\hline & & 141 & 9.38 & 17.72 & 8.34 & & 141 & 9.35 & 16.99 & 7.64 \\
\hline & & 142 & 8.93 & 17.03 & 8.10 & & 142 & 8.92 & 16.35 & 7.43 \\
\hline & & 143 & 8.49 & 16.33 & 7.84 & & 143 & 8.46 & 15.67 & 7.21 \\
\hline & & 144 & 8.07 & 15.67 & 7.60 & & 144 & 8.05 & 15.05 & 7.00 \\
\hline & & 145 & 7.68 & 15.05 & 7.37 & & 145 & 7.67 & 14.46 & 6.79 \\
\hline
\end{tabular}

Table 3. Bull call spread risk profile using Black-Scholes model.

\begin{tabular}{|c|c|c|c|c|c|}
\hline Step 1 & Long Call & & & & \\
\hline \multicolumn{6}{|c|}{ Buy R140 Strike Call@R11.77 } \\
\hline Step 2 & Short Call & Risk & Reward & Breakeven & Max ROI \\
\hline 1) & Sell R141 Strike Call@R10.54 & $\mathrm{R} 1.23$ & $-\mathrm{R} 0.23$ & $\mathrm{R} 141.23$ & $-18.70 \%$ \\
\hline 2) & Sell R142 Strike Call@R10.04 & $\mathrm{R} 1.73$ & $\mathrm{R} 0.27$ & $\mathrm{R} 141.73$ & $15.61 \%$ \\
\hline 3) & Sell R143 Strike Call@R9.59 & $\mathrm{R} 2.18$ & $\mathrm{R} 0.82$ & $\mathrm{R} 142.18$ & $37.61 \%$ \\
\hline 4) & Sell R144 Strike Call@R9.15 & $\mathrm{R} 2.62$ & $\mathrm{R} 1.38$ & R142.62 & $52.67 \%$ \\
\hline 5) & Sell R145 Strike Call@8.70 & R3.07 & R1.93 & R143.07 & $62.87 \%$ \\
\hline
\end{tabular}

Table 4. Bull call spread risk profile using VGSSD model.

\begin{tabular}{cccccc}
\hline Step 1 & Long Call & & & \\
\hline & Buy R140 Strike Call@R11.83 & & & & \\
\hline Step 2 & Short Call & Risk & Reward & Breakeven & Max ROI \\
$1)$ & Sell R141 Strike Call@R10.56 & R1.27 & - R0.27 & R141.27 & $-21.26 \%$ \\
$2)$ & Sell R142 Strike Cal1@R10.07 & R1.76 & R0.24 & R141.76 & $13.64 \%$ \\
$3)$ & Sell R143 Strike Cal1@R9.62 & R2.21 & R0.79 & R142.21 & $35.75 \%$ \\
$4)$ & Sell R144 Strike Call@R9.16 & R2.67 & R1.33 & R142.67 & $49.81 \%$ \\
$5)$ & Sell R145 Strike Call@R8.71 & R3.12 & R1.88 & R143.12 & $60.26 \%$ \\
\hline
\end{tabular}

a result choice 4) is attractive to create the strategy since the net credit is fairly high, and the breakeven point as well as the risk are reduced.

In Table 9 a potential strategy again can be created using a strike which provides reduced risk and lower breakeven. Also, the gain on this strategy is the net credit received upon entering the trade. As a result choice 4) is attractive to create the strategy since the net credit is fairly high, and the breakeven point is reduced and the risk is fairly low.

\section{3) Scenario Analysis at the Expiration Date}

Next, we look at the profit/loss of the strategy at expiration for a range of prices for the underlying using the choice selected in Step 2. We compare the profit/loss under the two models-Black-Scholes model and VGSSD model. Table 10 shows the profit/loss of the strategy under the 
Table 5. Bull call spread profit/loss under Black-Scholes and VGSSD models.

\begin{tabular}{cccc}
\hline Black-Scholes Model & & \multicolumn{2}{c}{ VGSSD Model } \\
\hline ASAQ@expiry & Profit/Loss & ASAQ@expiry & Profit/Loss \\
\hline 135 & -2.67 & 135 & -2.62 \\
136 & -2.67 & 136 & -2.62 \\
137 & -2.67 & 137 & -2.62 \\
138 & -2.67 & 138 & -2.62 \\
139 & -2.67 & 139 & -2.62 \\
140 & -2.67 & 140 & -2.62 \\
141 & -1.67 & 141 & -1.62 \\
142 & -0.67 & 142 & -0.62 \\
142.67 & 0.00 & 142.62 & 0.00 \\
143 & 0.33 & 143 & 0.38 \\
144 & 1.33 & 144 & 1.38 \\
145 & 1.33 & 145 & 1.38 \\
146 & 1.33 & 146 & 1.38 \\
147 & 1.33 & 147 & 1.38 \\
149 & 1.33 & 148 & 1.38
\end{tabular}

Table 6. Bear call spread investor choices.

\begin{tabular}{cll}
\hline Step 1 & Short Call & Sell R119 Strike Call \\
\hline Step 2 & 1) Long Call & Buy R120 Strike Call \\
Or & 2) Long Call & Buy R121 Strike Call \\
Or & 3) Long Call & Buy R122 Strike Call \\
Or & 4) Long Call & Buy R123 Strike Call \\
Or & 5) Long Call & Buy R124 Strike Call \\
\hline
\end{tabular}

Table 7. Bear call spread bid-ask prices at different stress levels.

\begin{tabular}{|c|c|c|c|c|c|c|c|c|c|c|}
\hline \multirow{2}{*}{$\begin{array}{l}\text { Stress } \\
\text { Level }\end{array}$} & \multicolumn{5}{|c|}{ Black-Scholes Model } & \multicolumn{5}{|c|}{ VGSSD Model } \\
\hline & $\mathrm{S}$ & $\mathbf{K}$ & Bid & Ask & Spread & $\mathrm{S}$ & $\mathbf{K}$ & Bid & Ask & Spread \\
\hline \multirow[t]{5}{*}{0.01} & 120 & 119 & 6.91 & 7.45 & 0.54 & 120 & 119 & 6.87 & 7.40 & 0.73 \\
\hline & & 120 & 6.40 & 6.90 & 0.50 & & 120 & 6.37 & 6.86 & 0.71 \\
\hline & & 121 & 5.92 & 6.40 & 0.48 & & 121 & 5.88 & 6.35 & 0.69 \\
\hline & & 122 & 5.47 & 5.93 & 0.46 & & 122 & 5.43 & 5.89 & 0.67 \\
\hline & & 124 & 4.62 & 5.03 & 0.41 & & 124 & 4.61 & 5.02 & 0.41 \\
\hline \multirow[t]{5}{*}{0.05} & 120 & 119 & 6.57 & 9.42 & 2.85 & 120 & 119 & 6.55 & 9.34 & 2.79 \\
\hline & & 120 & 6.06 & 8.76 & 2.70 & & 120 & 6.04 & 8.67 & 2.63 \\
\hline & & 121 & 5.61 & 8.16 & 2.55 & & 121 & 5.59 & 8.09 & 2.50 \\
\hline & & 122 & 5.18 & 7.61 & 2.43 & & 122 & 5.17 & 7.53 & 2.36 \\
\hline & & 124 & 4.35 & 6.50 & 2.15 & & 124 & 4.33 & 6.42 & 2.09 \\
\hline \multirow[t]{6}{*}{0.10} & 120 & 119 & 6.16 & 12.25 & 6.09 & 120 & 119 & 6.14 & 12.05 & 5.91 \\
\hline & & 120 & 5.67 & 11.44 & 5.77 & & 120 & 5.66 & 11.28 & 5.62 \\
\hline & & 121 & 5.25 & 10.74 & 5.49 & & 121 & 5.23 & 10.56 & 5.33 \\
\hline & & 122 & 4.81 & 10.00 & 5.19 & & 122 & 4.80 & 9.85 & 5.05 \\
\hline & & 123 & 4.42 & 9.33 & 4.91 & & 123 & 4.41 & 9.19 & 4.78 \\
\hline & & 124 & 3.71 & 8.08 & 4.37 & & 124 & 3.69 & 7.94 & 4.25 \\
\hline
\end{tabular}


Table 8. Bear call spread risk profile using Black-Scholes model.

\begin{tabular}{|c|c|c|c|c|c|}
\hline Step 1 & Short Call & & & & \\
\hline & Sell R119 Strike Call@R6.91 & & & & \\
\hline Step 2 & Long Call & Risk & Reward & Breakeven & Max ROI \\
\hline 1) & Buy R120 Strike Call@R6.90 & R0.99 & R0.01 & R119.01 & $1.01 \%$ \\
\hline 2) & Buy R121 Strike Call@R6.40 & $\mathrm{R} 1.49$ & $\mathrm{R} 0.51$ & R119.51 & $34.23 \%$ \\
\hline 3) & Buy R122 Strike Call@R5.93 & R2.02 & R0.98 & R119.98 & $48.51 \%$ \\
\hline 4) & Buy R123 Strike Call@R5.45 & $\mathrm{R} 2.54$ & $\mathrm{R} 1.46$ & R120.46 & $57.48 \%$ \\
\hline 5) & Buy R124 Strike Call@5.03 & $\mathrm{R} 3.12$ & $\mathrm{R} 1.88$ & R120.88 & $60.26 \%$ \\
\hline
\end{tabular}

Table 9. Bear call spread risk profile using VGSSD model.

\begin{tabular}{|c|c|c|c|c|c|}
\hline Step 1 & Short Call & & & & \\
\hline \multicolumn{6}{|c|}{ Sell R119 Strike Call@R6.87 } \\
\hline Step 2 & Long Call & Risk & Reward & Breakeven & $\begin{array}{l}\text { Max } \\
\text { ROI }\end{array}$ \\
\hline 1) & $\begin{array}{c}\text { Buy R120 Strike } \\
\text { Call@R6.86 }\end{array}$ & R0.99 & R0.01 & R119.01 & $1.01 \%$ \\
\hline 2) & $\begin{array}{c}\text { Buy R121 Strike } \\
\text { Call@R6.35 }\end{array}$ & $\mathrm{R} 1.48$ & R0.52 & R119.52 & $35.14 \%$ \\
\hline 3) & $\begin{array}{c}\text { Buy R122 Strike } \\
\text { Cal1@R5.89 }\end{array}$ & $\mathrm{R} 2.02$ & R0.98 & R119.98 & $48.51 \%$ \\
\hline 4) & $\begin{array}{c}\text { Buy R123 Strike } \\
\text { Call@R5.43 }\end{array}$ & $\mathrm{R} 2.56$ & $\mathrm{R} 1.44$ & $\mathrm{R} 120.44$ & $56.25 \%$ \\
\hline 5) & $\begin{array}{c}\text { Buy R124 Strike } \\
\text { Call@5.02 }\end{array}$ & R3.15 & $\mathrm{R} 1.85$ & $\mathrm{R} 120.85$ & $58.73 \%$ \\
\hline
\end{tabular}

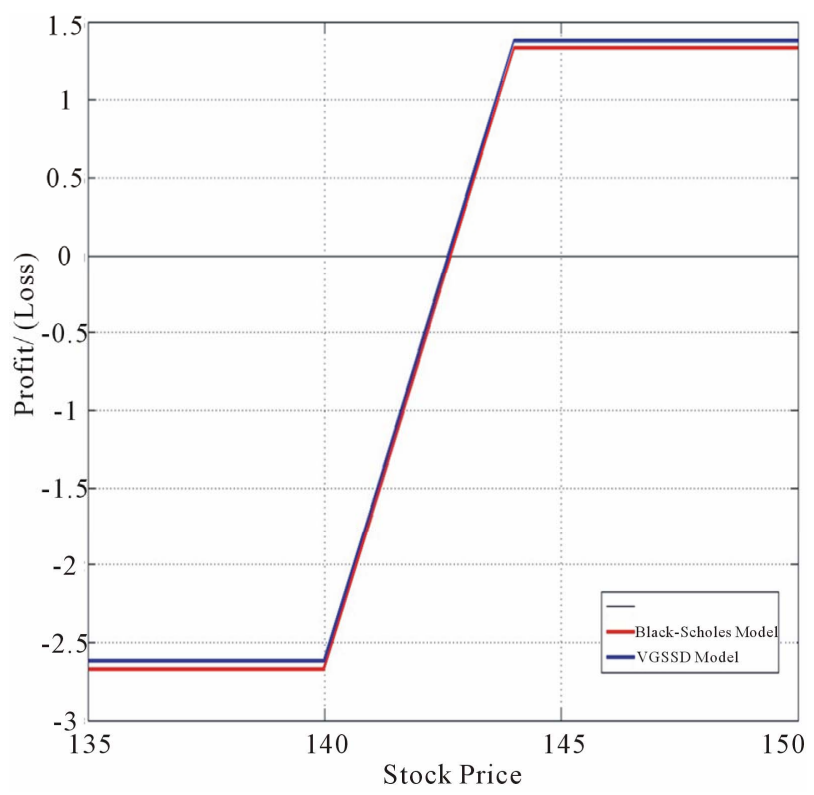

Figure 1. Plot of bull call spread profit/loss.

two models. Figure 2 shows the plot of the profit/loss of the strategy for a range of prices of the underlying at expiration. The breakeven point is lower in the VGSSD model than the Black-Scholes model, which is ideal in creating a strategy with reduced risk.
Table 10. Bull call spread profit/loss under the Black-Scholes and VGSSD models.

\begin{tabular}{cccc}
\hline \multicolumn{2}{c}{ Black-Scholes Model } & \multicolumn{2}{c}{ VGSSD Model } \\
\hline SBKQ@expiry & Profit/Loss & SBKQ@expiry & Profit/Loss \\
\hline 125 & -2.54 & 125 & -2.56 \\
124 & -2.54 & 124 & -2.56 \\
123 & -2.54 & 123 & -2.56 \\
122 & -1.54 & 122 & -1.56 \\
121 & -0.54 & 121 & -0.56 \\
120.46 & 0.00 & 120.44 & 0.00 \\
120 & 0.46 & 120 & 0.44 \\
119 & 1.46 & 119 & 1.44 \\
118 & 1.46 & 118 & 1.44 \\
117 & 1.46 & 117 & 1.44 \\
116 & 1.46 & 116 & 1.44 \\
115 & 1.46 & 115 & 1.44 \\
\hline
\end{tabular}

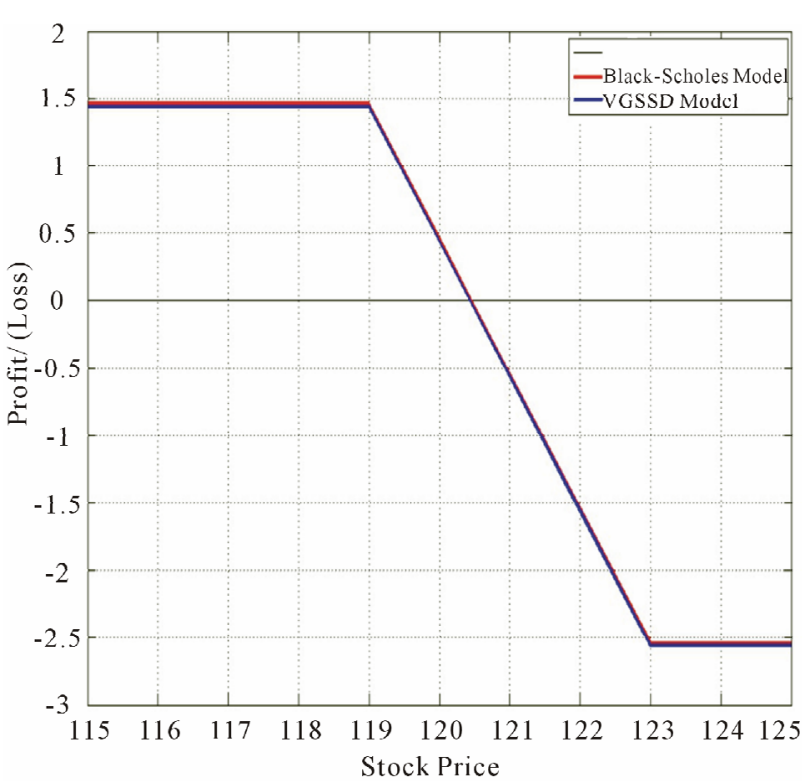

Figure 2. Plot of bear call spread profit/loss.

\subsubsection{Comment on the Strategy}

Under this strategy, the spread is reduced under the VGSSD model than the Black-Scholes model. The lower spread implies reduced cost of risk and lower breakeven 
point. However, in this strategy reducing the risk impacts on the potential reward under the VGSSD model as compared to the Black-Scholes model.

\section{Conclusions}

In this paper, we have looked at the quantification of risks of trading strategies in incomplete markets. We established that the no-arbitrage price intervals are unacceptably large. We need intervals with prices which are acceptable to the market. The acceptability of the prices is assessed by risk measures. Ideal risk measures are those that produce price bounds that are suitable for use as bid-ask prices and are able to compensate for unhedgeable risk. Plausible risk measures we look at are coherent risk measure and acceptability indices. Acceptability indices are heavily used in the theory of conic finance, which we used to assess the risk of trading strategies. Conic finance provides plausible bid-ask prices which are determined only by the probability distribution of the cash flow.

We assess the risks of financial positions using two strategies-bull call spread and bear call spread. Comparison of the risk profiles for the trading strategies is done between the VGSSD model and the Black-Scholes model. The findings showed that the spread was reduced, especially using the VGSSD model as compared to the Black-Scholes model. In addition, the findings showed that the bid-ask price intervals are able to compensate for the unhedgeable risk. Ultimately, reward from the strategies had a potential of increasing.

\section{REFERENCES}

[1] P. Artzner, F. Delbaen, J. Eber and D. Heath, "Definition of Coherent Measure of Risk," Mathematical Finance, Vol. 9, No. 3, 1999, pp. 203-228. http://dx.doi.org/10.1111/1467-9965.00068
[2] A. Cherny and D. Madan, "New Measure of Performance Evaluation," Review of Financial Studies, Vol. 22, No. 7, 2009, pp. 2571-2606. http://dx.doi.org/10.1093/rfs/hhn081

[3] J. N. Cochrane and J. Saá-Requejo, "Beyond Arbitrage: 'Good Deal' Asset Price Bounds in Incomplete Markets,' Journal of Political Economy, Vol. 108, No. 1, 2000, pp. 79-11. http://dx.doi.org/10.1086/262112

[4] J. Staum, "Incomplete Markets," In: J. R. Birge and V. Linetsky, Handbook in Operations Research and Management Science, Vol. 15, Chapter 12, Elsevier, Berlin, 2008, pp. 511-563.

[5] S. Jaschke and K. Küchler, "Coherent Risk Measures and Good Deal Bounds," Finance and Stochastics, Vol. 5, No. 2, 2001, pp. 181-200. http://dx.doi.org/10.1007/PL00013530

[6] A. Cherny and D. Madan, "Markets as a Counterparty: An Introduction to Conic Finance," International Journal of Theoretical and Applied Finance, Vol. 13, No. 8, 2010, pp. 1149-1177. http://dx.doi.org/10.1142/S0219024910006157

[7] D. Madan, P. Carr and E. Chang, "The Variance Gamma Process and Option Pricing," European Finance Review, Vol. 2, No. 1, 1998, pp. 79-105. http://dx.doi.org/10.1023/A:1009703431535

[8] P. Carr, H. Geman, D. Madan, and M. Yor, "Self Decomposability and Option Pricing," Mathematical Finance, Vol. 17, No. 1, 2007, pp. 31-57. http://dx.doi.org/10.1111/j.1467-9965.2007.00293.x

[9] K. Sato, "Self Similar Processes with Independent Increments," Probability Theory and Related Fields, Vol. 89, No. 3, 1991, pp. 285-300. http://dx.doi.org/10.1007/BF01198788

[10] K. Sato, "Lévy Processes and Infinitely Divisible Distributions," Cambridge University, Cambridge, 1999.

[11] P. Carr, H. Geman, D. Madan and M. Yor, "Pricing Options on Realized Variance," Finance and Stochastics, Vol. 9, No. 4, 2005, pp. 453-475. http://dx.doi.org/10.1007/s00780-005-0155-x 